Revista Facultad 2019

\title{
Malestar en el trabajo: una mirada a contadores públicos en farmacéuticas ${ }^{*}$
}

\author{
Jeimy Paola Pedraza Benítez ${ }^{\mathbf{a}}$ - Daimer Higuita-López
}

\begin{abstract}
Resumen: Este artículo aborda el malestar laboral en contadores públicos de Bogotá, en multinacionales del sector farmacéutico. Concretamente, el estudio se propuso, por un lado, identificar los factores que le crean malestar laboral a los contadores de estas compañías y, por el otro, analizar los efectos de dichos aspectos sobre la esfera anímica de los sujetos. Con ayuda de una encuesta, de entrevistas en profundidad y un grupo foco, la investigación arrojó que el malestar laboral es disparado por factores internos y externos a las empresas; por ejemplo, las políticas basadas en estereotipos o la normatividad que regula el ejercicio contable. Algunos de estos factores se asocian con las denominadas enfermedades de la excelencia, características de un entorno laboral altamente competitivo.
\end{abstract}

Palabras claves: Contadores; farmacéuticas; frustración; malestar; organización; sufrimiento.

Fecha de recibido: 3 de mayo de 2018 Fecha de aprobado: 21 de mayo de 2019

Cómo citar: Pedraza Benítez, J. P. e Higuita-López, D. (2019). Malestar en el trabajo: una mirada sobre contadores públicos en farmacéuticas. Revista Facultad de Ciencias Económicas, 27(2), 67-82. rev.fac. cienc.econ. doi: https://doi.org/10.18359/rfce.3444

JEL: M49, M54.

* Artículo de investigación.

a Contador Público de la Universidad Central, Colombia, y magíster en Administración de la Universidad Nacional de Colombia. Correo electrónico: jeimypaolap@yahoo.com

b Ph.D. en Ciencias Económicas de la Universidad Nacional de Colombia, magíster en Ciencias de la Administración de la Universidad EAFIT, Colombia, y administrador de empresas de la Universidad de Antioquia, Colombia. Profesor de la Universidad Nacional de Colombia. Teléfono: (574) - 4255291. Correo electrónico: dhiguitalo@unal.edu.co 


\title{
Discomfort at Work: A Look at Public Accountants in Pharmaceutical Companies
}

\begin{abstract}
This paper discusses discomfort at work among public accountants in Bogotá in multinationals companies of the pharmaceutical sector. Specifically, on the one hand, the study was intended to identify factors that generate discomfort at work among accountants from these companies and, on the other hand, to analyze the impact of these aspects on the state of mind of the subjects. Using a survey, in-depth interviews and a focus group, research showed that the discomfort at work is triggered by the company's internal and external factors; for example, policies based on stereotypes or regulations that govern accounting practice. Some of these factors are associated with the so-called diseases of excellence common in a highly-competitive work environment.
\end{abstract}

Keywords: Accountants; pharmaceutical companies; frustration; discomfort; organization; suffering.

\section{Desconforto no trabalho: um olhar sobre contadores públicos na indústria farmacêuticos}

Resumo: Este artigo trata do desconforto que contadores públicos de Bogotá sentem no trabalho, em multinacionais do setor farmacêutico. Especificamente, o estudo propôs, por um lado, identificar os fatores que geram esse desconforto nos contadores dessas empresas e, por outro, analisar os efeitos desses aspectos na esfera psíquica dos sujeitos. Com a ajuda de enquetes, entrevistas detalhadas e um grupo de focal, a pesquisa mostrou que o desconforto no trabalho é desencadeada por fatores internos e externos às empresas, por exemplo, políticas baseadas em estereótipos ou nos regulamentos que regem o exercício contábil. Alguns desses fatores estão associados às chamadas doenças de excelência, características de um ambiente de trabalho altamente competitivo.

Palavras-chave: Contadores; farmacêuticas; frustração; desconforto; organização; sofrimento. 


\section{Introducción}

Atraemos alto desempeño, personas que son apasionadas de su carrera y tienen el deseo de superar las expectativas

Desde la aparición de la denominada escuela de relaciones humanas, se viene diciendo que los recursos humanos son uno de los elementos más importantes de las organizaciones, si no el más importante, y si el factor humano es importante, será necesario brindarle condiciones de bienestar laboral. Por eso, se han diseñado prácticas de gestión humana, beneficios extralegales y programas de bienestar, para reforzar la idea de que las personas son el recurso fundamental en una organización (Barrios e Illada, 2013). No obstante, Adecco (2014) expuso el resultado de un estudio realizado con 1255 trabajadores colombianos, que señala alertas para las compañías sobre el tema de bienestar, ya que las políticas en este terreno no parecen ser efectivas para el $40 \%$ de los encuestados.

Por otro lado, y aunque las organizaciones promueven la satisfacción laboral y el bienestar, también pueden ser productoras de malestar o insatisfacción, como lo evidencia el sondeo publicado por Montes (2019). En este se sugiere que ocho de cada diez colombianos están insatisfechos en su empleo; sin embargo, las empresas poco hablan del tema, tal vez porque se ha construido el imaginario de que sentirse incómodo, estresado o angustiado en el trabajo es una señal de ineficiencia o ineptitud en el empleado. Es decir, pareciera que existe la idea de que si la empresa promueve el bienestar y beneficio del trabajador, y aun así este se siente incómodo o estresado, entonces el problema está en el empleado y no en la organización.

No obstante, hoy se conocen casos de empresas donde se observan humillaciones y amenazas a los empleados, o prácticas que inducen al error, lo que crea desconcierto y confusión (Linhart, 2016). Son organizaciones que dan órdenes contradictorias, inviables o confusas, o exigen tareas descomunales (Blein Cabestrero, 2010). En diversas ocasiones los profesionales, entre ellos los contadores, no son respetados, valorados, ni suficientemente reconocidos por la empresa, incluidas las farmacéuticas. Para Morales (2007), estas amenazas, órdenes y poco reconocimiento provocan consecuencias como baja autoestima, cansancio, estrés y malestar general.

En su magnífico texto, Johnny Javier Orejuela (2018) señala estudios sobre el malestar en distintos grupos ocupacionales, como profesionales de la salud, trabajadores bancarios, operadores de call center, psicólogos e incluso pilotos. Sánchez Llull, March Cerdà y Ballester Brage (2015) con su trabajo realizado en Chile encuentran niveles significativos de burnout en profesores de los institutos de secundaria: un porcentaje importante de profesores y profesoras son susceptibles de desarrollar la enfermedad o ya la sufren (33\% aproximadamente). Ávila Toscano, Gómez Hernández y Montiel Salgado (2010) encuentran que la restricción de tiempo y las pocas posibilidades para interactuar limitan el reconocimiento y generan malestar en empleados de la salud. Igualmente, se ha encontrado que el reconocimiento excesivo de la jerarquía y de los resultados medibles transforma el trabajo en una práctica instrumental, que aleja la posibilidad de disfrutar de este (Guerrero Morales, Balboa Gallardo y Miranda Hiriart, 2017).

En el contex to latinoamericano viene tomando mayor fuerza la mirada de los investigadores sobre el malestar (Orejuela, 2018), aspecto que incluso ha motivado varias tesis doctorales en Colombia. No obstante, este es un tema de larga tradición investigativa en países como Francia, con Christoph Dejours y Vincent de Gaulejac. El malestar estudiado en esta investigación no es una incomodidad que imposibilita las tareas del contador; no es un malestar que envía a la clínica a los contadores, aunque en periodos crónicos de crisis nerviosas o de estrés sí se puede presentar esta situación (Aubert y De Gaulejac, 1991). Es un malestar que termina siendo parte de la función del contador; aun así, sigue siendo malestar. Por otro lado, el sector farmacéutico, además de ser un sector de crecimiento, tiene algunas especificidades importantes. Por ejemplo, la mayoría son empresas de carácter multinacional donde muchas de sus políticas son inspiradas en una casa matriz; algunas de esas políticas, como veremos, son uno de los factores internos generadores de malestar. Ahora bien, aunque algunos factores son identificados 
como externos a las organizaciones, por ejemplo, la normativa tributaria, estas organizaciones deben cumplir con protocolos y fechas establecidas en el país donde tengan domicilio. De esta manera, dado que algunos trabajos se han focalizado en las consecuencias sobre aspectos físicos y fisiológicos (Confederación Regional de Organizaciones Empresariales de Murcia [Croem], 2010; Unión General de Trabajadores [UGT], 2014, Loaiza Betancur, 2014), esta investigación se propuso identificar los factores que producen malestar y analizar los efectos de dichos factores sobre la esfera anímica de los contadores que se desempeñan en farmacéuticas.

Así, entonces, el documento se ha dividido en cuatro partes. La primera presenta los aspectos preliminares de la investigación; la segunda expone brevemente un análisis teórico sobre causas y consecuencias del malestar laboral; la tercera traza el camino metodológico, realizado con una mirada comprensiva (este trabajo de terreno se hizo con contadores públicos que laboran en Bogotá, en compañías farmacéuticas), y la cuarta expone los resultados del estudio y sus conclusiones.

\section{Rasgos de una realidad organizacional}

El malestar que en épocas contemporáneas viven los empleados cobra cada vez mayor fuerza. En su clásico Le coût de l'excellence (1991), Aubert y De Gaulejac exponen que el malestar (y algunas enfermedades psíquicas) es inducido por diversas organizaciones que, persiguiendo la excelencia, incitan a los trabajadores a un reto constante de superación. Y esta lógica persiste en la actualidad. Por ejemplo, una de las farmacéuticas abordadas en esta investigación menciona: "[...] se trata de que todos se esfuercen para impactar los resultados [...] este mes, este trimestre, este año y durante muchos años [...] en el futuro" (farmacéutica P, 2015) ${ }^{1}$.

Después de analizar una encuesta aplicada a 55 contadores públicos del sector farmacéutico, se encontró que los resultados operacionales de la empresa no se corresponden con las condiciones

1 Para mantener la confidencialidad, los nombres de las compañías se designarán con una letra. La información fue tomada de la web de la compañía. de trabajo. En otras palabras, aunque la empresa obtiene muy buenos resultados, las condiciones laborales no mejoran y son percibidas de manera negativa por los trabajadores. Además, muchos contadores manifiestan síntomas como depresión o tristeza en el trabajo.

Así, pues, además de la idea de bienestar, "el malestar" se ha constituido igualmente en tema de estudio en las organizaciones, y ha ganado relevancia en los últimos años (Dejours, 2003; De Gaulejac, 2011; Han, 2012; López, 2014).

Desde algunas miradas reflexivas, se dice que el mundo laboral es víctima de una cadena que finaliza con el detrimento de los trabajadores (Gómez Vélez y Calderón Carrascal, 2017). También se ha dicho que el malestar laboral es uno de los causantes de las enfermedades cardiovasculares, así como de aquellos males conexos a la salud mental, por ejemplo, ansiedad o depresión (Croem, 2010). A partir del momento cuando la lógica financiera ha dominado la lógica de producción, el trabajo se ha tornado en una fuerte presión de los inversionistas (Dériot, 2010). Pero, ¿qué puede entenderse por malestar?

El término "mal", del latín male, se refiere al perjuicio o agravio que recibe una persona; está asociado a calamidad, desgracia, dolencia, enfermedad. El término "estar", del latín stare, significa existir, hallarse en un lugar, situación, condición o modo actual de ser. El malestar puede entenderse como la sensación de inquietud y desazón que se siente ante un acontecimiento considerado de forma negativa (Abbagnano, 2010). En particular, el ejercicio de la profesión contable en Colombia parece estar acompañado de esa desazón, pues se desarrolla bajo condiciones extremas de carga física y mental (Loaiza Betancur, 2014).

El malestar es un periodo difícil durante el cual un individuo no se siente bien. Este sentimiento tiene origen físico o psicológico. Aunque ese momento puede ser temporal, también es posible que sea un indicador de una patología o de situaciones como depresión. Para Freud (1988), el malestar es "un sufrimiento cuyo origen puede surgir del cuerpo, desde el exterior y de la relación entre las personas" (p. 85). En la tabla 1 se presenta sucintamente la distinción entre malestar, sufrimiento y dolor psíquico. 
Tabla 1. Continuidades entre las nociones malestar, sufrimiento y dolor psíquico

\begin{tabular}{llllll}
\hline & Intensidad & Cualidad & Simbolización & Tolerancia \\
\hline Malestar & Baja & Difuso & Se puede o no articular & Tolerable \\
\hline Sufrimiento & Alta & Relativamente identificable & Sin sentido, inarticulable & Intolerable \\
\hline Dolor psíquico & Media/alta & Claramente identificable & Articulable. & Tolerable \\
\hline
\end{tabular}

Fuente: Orejuela (2018, p. 108).

\section{Causas del malestar laboral}

Las empresas incitan a la persona a alcanzar la excelencia, y para esto le proponen medir su realización personal en función de los resultados financieros de la firma (Aubert y De Gaulejac, 1991). En esta lógica, el trabajador no se siente explotado, pues este es persuadido por la oportunidad de gobernar sus quehaceres o "convertirse" en su propio jefe. De este modo, otra farmacéutica declara: "atraemos alto desempeño, personas que son apasionadas de su carrera y tienen el deseo de superar las expectativas" (farmacéutica A, 2015).

El informe de malestar laboral realizado por la Comisión de Asuntos Sociales de París (Dériot, 2010) propone distintas causas (internas y externas) del malestar laboral. Las causas internas a la organización están asociadas al trabajador encerrado en su trabajo, al aislamiento, al desempeño a cualquier precio, a la pérdida de compromiso por el trabajo y las malas condiciones físicas y técnicas. Las causas externas a la organización son el estrés en el transporte y la doble responsabilidad que afrontan las mujeres como amas de casa y trabajadoras.

El trabajador encerrado en su trabajo. Si los empleados están más comprometidos con la idea de carrera dentro de la organización se sienten encerrados en su trabajo (Tisnés, Lopera, Pérez, Ramírez y Henao, 2015). Esto significa que en algunos casos los empleados son, en cierto modo, "prisioneros" de su contrato de trabajo. Una consecuencia de esto es que cuando se sienten agredidos no responden, porque consideran que ese comportamiento puede ser visto como un indicador de incompetencia profesional o porque pueden ser despedidos. Parece persistir la siguiente idea: dado todo lo que la empresa hace por el bienestar y beneficio del trabajador, este no responde porque cree que puede ser despedido, ya que considera que el problema está en él mismo y no en la organización.

El aislamiento. Hoy se observan unas prácticas de gestión que favorecen el individualismo por encima del trabajo colectivo; como consecuencia, la lucha por los puestos de trabajo o por ascensos puede deteriorar la solidaridad en el interior de una misma clase social (Aubert y De Gaulejac, 1991; Bermúdez, 2017). En la sociedad contemporánea los sujetos luchan, de forma individual, por sus intereses, y esto aviva el aislamiento dentro de la empresa. Ese aislamiento es una fuente latente de tristeza y depresión (Benaiges, 2012).

El desempeño a cualquier precio. El éxito de la empresa y de la persona son el objetivo principal, de tal forma que el empleado interioriza los valores, los objetivos y la filosofía de la empresa (Aubert y De Gaulejac, 1991). Para lograr esto, las empresas usan estrategias de adhesión de tipo financiero y espiritual. En el primero encontramos casos como: "Nuestro objetivo es ofrecer un paquete retributivo de salario y beneficios sociales atractivo y competitivo para nuestros empleados" (farmacéutica T, 2012). En el segundo se fomenta la idea de grupo o se alude a la vida personal: "Brindamos conferencias para favorecer el equilibrio vida-trabajo en cada uno de los trabajadores" (farmacéutica P, 2015).

La pérdida de interés por el trabajo. Es la cuarta causal interna del malestar laboral. La búsqueda de la exigencia y la lucha para triunfar son consecuencia de la internalización de los objetivos y valores de la organización. Desde este sistema, se pide al individuo que haga cada día más, que se supere y sobrepase así lo que se le exige (Aubert y De Gaulejac, 1991). Dado que muchas compañías exigen plazos cortos de entrega para los bienes ofrecidos, en algunos casos esa exigencia se traduce en bienes de menor calidad, lo que activa malestar laboral en 
las personas, representado en el sentimiento de incomodidad y frustración por realizar un trabajo de baja calidad (Dériot, 2010).

Las condiciones técnicas y físicas del lugar de trabajo. Son otra causal de malestar laboral y hacen referencia a los atributos de los equipos, las instalaciones y demás elementos necesarios para ejecutar un rol.

Adicional a las causas mencionadas, existen otras que han sido consideradas externas a la organización, como el estrés en el transporte, factor que ha sido ampliamente estudiado por La Chaire Management et Santé au travail, en Grenoble, Francia $^{2}$. En el caso colombiano, las pésimas circunstancias del transporte en Bogotá y el tiempo para llegar de la casa al trabajo son otra fuente de malestar laboral. Estas circunstancias crean sufrimiento y angustia. En este caso, los inconvenientes frecuentes son "el hacinamiento, los retrasos por el tráfico, el sentimiento de pérdida de tiempo en el transporte y la fatiga" (Semana, 2014).

La idea de doble jornada femenina está asociada con el hecho de que las mujeres deben alternar el trabajo en el hogar con sus funciones en la empresa, y aunque esta situación ha sido naturalizada por la mayoría de las mujeres, debe ser considerada como uno de los problemas principales de la mujer actual (Fernández Collados, 2012). Aquí es importante una precisión: no se está afirmando que solamente existan estas siete causas del malestar, sino que son las que, dado el interés del estudio, arrojó la revisión de la literatura. En cualquier caso, estas condiciones (internas y externas) pueden tener consecuencias a nivel físico, pero sobre todo afectan mental y socialmente a los trabajadores (Marulanda, 2007).

En la tabla 2 se sintetizan estas causas con sus respectivos síntomas.

Como se puede observar en la tabla 1, el malestar en el trabajo afecta tanto la parte física del ser humano, como sus esferas espiritual, emocional y mental. Esto ha contribuido a usar la expresión estrés de origen laboral, del cual se hablará en el apartado "La normativa".

2 Ver, por ejemplo, Rossano, Chatillon y Desmarais, 2015).
Tabla 2. Causas-efectos del malestar laboral

\begin{tabular}{|c|c|c|}
\hline 莺 & Síntomas & Efectos \\
\hline \multirow{4}{*}{ 冚 } & $\begin{array}{l}\text { El desempeño a cualquier } \\
\text { precio. }\end{array}$ & Angustia, estrés. \\
\hline & El aislamiento. & Depresión, tristeza. \\
\hline & $\begin{array}{l}\text { La pérdida de compromiso } \\
\text { por el trabajo. }\end{array}$ & $\begin{array}{l}\text { Sufrimiento, fracaso, } \\
\text { angustia, Incomodidad. }\end{array}$ \\
\hline & $\begin{array}{l}\text { El trabajador encerrado en } \\
\text { su trabajo. }\end{array}$ & Miedo. \\
\hline \multirow{3}{*}{ 军 } & El estrés en el transporte. & $\begin{array}{l}\text { Sufrimiento, angustia, } \\
\text { fatiga, agresividad, } \\
\text { dolencias físicas, estrés. }\end{array}$ \\
\hline & $\begin{array}{l}\text { Las malas condiciones } \\
\text { físicas y técnicas. }\end{array}$ & $\begin{array}{l}\text { Incomodidad, molestias, } \\
\text { dolencias físicas, trastornos } \\
\text { psicosociales. }\end{array}$ \\
\hline & $\begin{array}{l}\text { La doble jornada de la } \\
\text { mujer (profesional/ama } \\
\text { de casa). }\end{array}$ & Cansancio, fatiga, estrés. \\
\hline
\end{tabular}

Fuente: elaboración propia, a partir de Pedraza (2016) y Dériot (2010).

Las condiciones de trabajo propiciadoras de malestar laboral, planteadas por la UGT de España (2014) y por la Comisión de asuntos sociales de París (Dériot, 2010), son generales y no especifican roles particulares, como el caso de los contadores. Por tanto, es necesario precisar que, como otros trabajos han abordado la salud del trabajador en sus aspectos físicos y fisiológicos (Croem, 2010; UGT, 2014; Loaiza Betancur, 2014), esta investigación se focalizó en una población específica (contadores que laboran en farmacéuticas en Bogotá) para identificar factores que producen malestar y analizar los efectos en el aspecto anímico de estos trabajadores; esto, sin desconocer la interdependencia entre lo físico, lo psíquico y lo social, en cada ser humano.

\section{Metodología}

Para delimitar la población en este estudio se consideraron tres criterios. Primero, escoger una profesión que presentara situaciones de malestar laboral; de acuerdo con Loaiza Betancur (2014), el ejercicio de la contaduría en Colombia presenta 
estas situaciones. Adicionalmente, en esta profesión se ha observado poco reconocimiento del esfuerzo, alto volumen de trabajo y jornadas laborales intensas (Soler, 2008). Segundo, el sector farmacéutico en Colombia se considera que tiene buenos salarios y que exige gran competencia a sus miembros (El Espectador, 2015), como se vio en lo que expresaron las compañías $\mathrm{P}, \mathrm{T}$ y A. Tercero, para determinar el grupo de personas objeto de estudio, se tuvo en cuenta el resultado de la encuesta realizada a 55 contadores públicos que trabajan en el sector farmacéutico, los cuales manifiestan síntomas de malestar laboral. Con estos criterios, se dio inicio al trabajo de campo para identificar factores (el qué), sus indicadores (el por qué) y sus efectos.

\section{Exploración}

El trabajo sobre el terreno se desarrolló en tres momentos, de acuerdo con la metodología propuesta por (Galeano, 2004) exploración, focalización, profundización. Para cada momento se empleó una herramienta de producción de la información: encuesta, entrevista y grupo focal.

El primer acercamiento con los contadores se realizó a través de una encuesta, orientada a identificar los factores que le crean malestar laboral a los contadores de estas compañías. En concreto, la encuesta preguntaba por el qué (que hace alusión a los factores); adicionalmente, y de forma exploratoria, se indagaba por algunos de los síntomas presentados en la población. El criterio utilizado fue la oportunidad en la respuesta: se envió el cuestionario por correo electrónico y se definió una fecha de cierre, a la cual 55 contadores respondieron.

A partir de los resultados que arrojó esta encuesta, se descartaron los factores asociados a lo físico o lo fisiológico, pues no era propósito del estudio ${ }^{3}$.

\section{Focalización}

En esta etapa se utilizó la entrevista para focalizar la indagación sobre seis factores como posibles

3 No confundir los efectos en la salud física con las condiciones físicas del trabajo, consideradas como una de las causas de malestar laboral. Ahora bien, esas condiciones físicas podrían, igualmente, generar efectos en lo anímico. causantes de malestar laboral, los cuales se exponen en la sección de resultados. El instrumento estuvo dirigido a 16 contadores que trabajan para el sector farmacéutico en Bogotá, quienes voluntariamente decidieron participar. Al final de la encuesta se les solicitaba enviar un correo que indicara si deseaban ser entrevistados. Esta etapa indagaba por qué esos factores son causantes de malestar laboral; es decir, se buscaba rastrear indicadores de la manera como los factores afectan a los contadores. En cada empresa se entrevistó a dos contadores, escogidos por su disposición para participar. Son empresas que tienen más de cinco años en el país y son consideradas multinacionales.

Por su parte, los contadores tienen entre 29-35 años y poseen títulos de posgrado. Cuentan con al menos cuatro años de trayectoria en este sector, realizando labores en el tema financiero; además, se caracterizan por haber sido promovidos en las empresas donde trabajan.

Cada entrevista tardó 35 minutos, tiempo durante el cual el entrevistado, mediante el diálogo abierto, podía expresar sus vivencias respecto al malestar laboral. Para cada factor propiciador de malestar laboral se hicieron preguntas como: ¿cuál es su sensación, sentimiento, respecto a esos factores o condiciones? ¿Cómo lo manejan en la empresa para la cual trabajan? ¿Por qué es causante de malestar laboral dicho factor? Todas estas preguntas estuvieron orientadas a la especificidad del rol contable en Colombia.

\section{Profundización}

El propósito fue analizar los efectos que dichos factores tienen sobre la esfera anímica de los sujetos-contadores. Para ello, se analizó la información proveniente de las entrevistas, con el fin de asociar y relacionar los resultados, en términos de factores y sus indicadores. En la sección de resultados se presenta gráficamente esta asociación.

Una vez se tenían estas asociaciones (de factores con sus indicadores), se llevó a cabo un grupo focal, en el que participaron ocho contadores, y los criterios para ser parte del grupo fueron: deseo de participar, experiencia en el área financiera y espontaneidad en la entrevista. 
Se optó, entonces, por el grupo foco, ya que permite verificar la validez de la información en el mismo momento que se está recolectando (Bonilla y Rodríguez, 2013). Es decir, el propósito del grupo foco fue validar la información recabada en las entrevistas. Por eso, para cada factor y sus indicadores se discutieron los efectos sobre la vida anímica de los contadores. Este ejercicio tardó 120 minutos aproximadamente.

\section{Análisis de resultados}

Los contadores participantes en la investigación confirmaron sentir malestar laboral derivado de la evaluación del rendimiento, el estatus social forjado por la empresa, los comportamientos del jefe, las políticas asentadas en estereotipos, la normatividad y desterritorialización. A continuación se exponen estos factores, así como sus indicadores y sus efectos sobre el estado anímico. Las expresiones de los contadores aparecen en cursiva.

\section{Evaluación de desempeño}

La evaluación del desempeño es un proceso que busca medir el trabajo y los resultados de los empleados. Medir el desempeño de un trabajador implica evaluar los índices de sus responsabilidades y funciones, así como los logros alcanzados en su cargo, de acuerdo con los objetivos fijados por la empresa y por un tiempo específico. Luego, se da a conocer al trabajador evaluado cuáles son sus debilidades y fortalezas, de manera que se pueda diseñar un plan de mejoramiento (Gerencia, 2012). Pero, ¿por qué la evaluación del rendimiento es un factor de malestar laboral?

Esta evaluación de desempeño crea sentimientos de intimidación o de frustración, cuando los empleados sienten que no se logran los objetivos, esos que desde el planteamiento se pueden considerar inalcanzables. Además, la evaluación de desempeño ha sido vista como una calificación del jefe, en ejercicio de su poder, en el que, en ocasiones, se expresa explícitamente que de ella depende la continuidad en la organización. De igual manera, durante la evaluación el evaluador no puede ser muy flexible, sino que tiene que aplicar un instrumento; pero dicho instrumento no siempre está adaptado a las condiciones del evaluado (Rodríguez, De Freitas y Zaá, 2012).

Por otro lado, para los participantes del estudio, la evaluación de desempeño es un proceso abrumador porque el área financiera tiene una gran responsabilidad en el primer trimestre de cada año, y es en ese momento cuando se trazan los objetivos. Un contador manifiesta:

Si te trazas esos objetivos, te pones las metas que vas a cumplir. Sin embargo, durante esta época del año este proceso es caótico. Tú no sabes ni siquiera por dónde empezar, entonces llega la angustia y también el estrés, por la competencia que se genera con los demás compañeros.

Asimismo, las empresas aspiran a que la evaluación de desempeño se constituya en una evaluación integral; es decir, que incluya los objetivos personales y los profesionales. No obstante, los contadores ven una contradicción en dicha aspiración. Primero, porque para ellos la esfera personal es precisamente "personal", y en consecuencia no desean revisar su proyección personal con el jefe; segundo, si deben cumplir unas metas profesionales tan altas, ¿a qué horas pueden cumplir los propósitos personales?

Esos visos contradictorios y saber que de la evaluación depende la continuidad en el trabajo son fuente de angustia y de incertidumbre; una angustia que, al ser generada en una relación de autoridad, da cuenta a su vez de la idea de dominación. No solo hay que cumplirle a la empresa con sus objetivos, sino además con nuestros objetivos personales.

Los contadores también señalan que para alcanzar los objetivos de sus áreas han sacrificado tiempo con su familia, o incluso otras diligencias personales: actividades como reuniones familiares, deporte, anulación de citas médicas, entre otros. Estas formas de medición y sus correspondientes indicadores también son causantes de malestar en los docentes estudiados en Chile (Guerrero et al., 2017).

La evaluación de desempeño en estas farmacéuticas busca que cada persona realice un trabajo extra, el cual debe completarse después de las tareas diarias. Esto implica ejecutar labores en 
horarios adicionales y con base en ese trabajo extra se califica su rendimiento. Estas circunstancias activan el estrés laboral en las personas, pues además del volumen de trabajo diario, cuando se dan cuenta se ha terminado el año y no han cumplido los objetivos. Esto favorece estados de frustración $o$ angustia.

De acuerdo con lo anterior, puede decirse que, a diferencia de otros roles donde la angustia se puede derivar del temor a no cumplir un número o un indicador, en los contadores la evaluación es un factor de malestar porque a veces se plantean objetivos inalcanzables, se utiliza como un ejercicio de poder, los objetivos se trazan en el periodo más difícil para su rol, se encuentran una contradicción respecto a que se evalúen objetivos personales y organizacionales, y porque deben sacrificar tiempo y actividades personales. Adicionalmente, la evaluación de desempeño lleva a los contadores a castigarse para alcanzar el estándar de estas organizaciones, que viven en una constante búsqueda de rentabilidad y les remarcan que el tiempo para lograr sus objetivos personales es reducido. Por todo esto, ese proceso se considera frustrante, a la vez que genera angustia por perder el trabajo. Pensar en la evaluación de desempeño causa fatiga emocional y, en ocasiones, hace que se renuncie a los tan ansiados objetivos profesionales (Loaiza Betancur, 2014).

\section{Ejercicio de autoridad de los jefes}

Cuando las acciones de los jefes (inmediatos o no) están orientadas a presionar por indicadores, puede decirse que el estilo de liderazgo genera malestar laboral en los individuos, ya que estos trabajan en pro de obtener resultados, lo que limita el conocimiento y la creatividad del empleado. Las personas se sienten resentidas al ser tratadas de esta manera (Naranjo, 2012). Pero lo particular en el caso de los contadores es que se escucha la frase "lo hicimos bien" cuando se cumplen los objetivos, pero en el caso contrario, cuando no se alcanzan las metas, se escucha: "usted lo hizo mal".

Por momentos, el jefe le hace creer al trabajador que la compañía busca su desarrollo personal para que este se esfuerce, cuando lo que quiere es maximizar los resultados de la organización, y el trabajador termina sintiéndose utilizado. Para algunos jefes de estas farmacéuticas, pareciera que el contador es una cosa de la cual disponen libremente, y esa percepción que tienen de él se materializa con órdenes que dan cuenta de su abuso de poder. $\mathrm{Al}$ respecto, un entrevistado comenta:

El jefe siempre quiere reunirse conmigo a la hora de mi almuerzo, y debo estar disponible hasta que él se vaya. Algunas veces me ha hecho quedar hasta las 8:00 p. m. Esa situación constituye para mí abuso de poder, lo que me ha afectado emocionalmente, pues siento que no tengo horario.

En muchos casos, con políticas de bonificaciones y aplausos, los jefes fomentan la competencia entre colegas, lo cual crea, además de estrés y ansiedad, algunas prácticas desleales que traen consecuencias en el ambiente laboral, la salud y la productividad (Loaiza Betancur, 2014). Para reforzar esa competencia, aparecen frases recurrentes en los jefes como: "Trabaje como si esta fuera su propia empresa", "usted es su propio jefe", "no haga las cosas por obligación", "hágalo porque usted lo siente”, "póngase la camiseta”, "si la empresa gana, ganamos todos".

Obsérvese cómo la estrategia discursiva del jefe busca persuadir al trabajador de que los logros de la compañía son sus propios logros. El jefe trata de influir en las ideas del contador, y para ello le promete seguridad laboral, cuando en realidad son logros ajenos a sí mismo. Y por buscar lo uno, se renuncia a lo otro o se pierde la libertad. Así, como sostienen Bauman y Dessal (2014), "las aflicciones y los malestares psicológicos provienen en su mayoría de la renuncia a una considerable porción de libertad a cambio de un incremento en la seguridad" (p. 19). De manera que el ejercicio de autoridad del jefe de la farmacéutica es un factor generador de malestar laboral por presionar por indicadores, porque a veces el contador se siente utilizado, porque abusa de su poder y porque fomenta la competencia entre los empleados. Esto trae como resultado prácticas desleales entre los empleados. Es, en suma, un ejercicio de autoridad que, aunque revestido de la ilusión de que no existen distancias jerárquicas (usted es su propio jefe), dan cuenta de dominación. Dominación que es disfrazada por el jefe con la idea 
de "libertad" (Han, 2012). Y el contador para escapar y no sentirse utilizado o presionado se enfrenta a la disyuntiva: se va o se "siente" libre.

\section{Estatus social generado por la empresa}

El estatus es sinónimo de prestigio y posición social, y puede ser determinado por el origen, el sexo, la clase social, la edad o la profesión. En esta investigación nos referimos a la profesión. El estatus puede ser adscrito o adquirido. El primero es una posición social que se obtiene al nacer, mientras que el segundo se refiere a aquellas posiciones que se pueden lograr a través del esfuerzo personal de los individuos (Ceballos, 1997).

En varias farmacéuticas se promueve el estatus como premio por la buena evaluación y, en esa medida, se dan a los empleados beneficios o recompensas de acuerdo con su ubicación en la jerarquía organizacional; beneficios como asistentes, oficinas grandes, parqueaderos reservados. Así, con estas ayudas para el individuo, las empresas refuerzan la idea y la importancia del estatus (Fischman, 2015). Sobre el particular, un entrevistado expresa:

Tu forma de ser, de vestir, "debe adaptarse" para poder llegar a ciertas posiciones. Pero para que te sientas parte del grupo debes compartir características en común, que al final te generan malestar porque no estás siendo auténtico. Y esto a largo plazo crea sentimientos de dependencia que te hacen sentir miedo de perder tu trabajo.

En estas empresas multinacionales, los contadores se ven inmersos en una competencia para llegar a ser parte del selecto grupo que respira excelencia. Para Bauman y Dessal (2014), “donde hay coacción, es decir donde las personas se ven obligadas a mantener un comportamiento diferente del que dictan sus inclinaciones naturales, hay descontento y disenso: la mayor parte del tiempo, sofocados, reprimidos o desviados, pero manifiestos de tanto en tanto" (p. 30).

La empresa de consultoría Fischman y Asociados (Fischman, 2015) indicó que los trabajadores le temen a la evaluación de desempeño porque ven su estatus en riesgo. Como se dijo con anterioridad, durante la evaluación de desempeño existe una etapa de retroalimentación, en la cual el responsable del área le indica al trabajador sus posibilidades de mejoramiento. Sin embargo, el trabajador, de acuerdo con la consultora, siente miedo a perder los beneficios que ha adquirido gracias a su estatus dentro de la organización.

Este estatus promovido por las farmacéuticas (muy propio de ellas y de las multinacionales) contribuye al malestar laboral, ya que ponen al contador en una lucha por alcanzar el estatus social y a hacer lo necesario por mantenerse en ese estatus. Sus acciones, profesionales o personales, determinarían su permanencia en la empresa. En este sentido, Bauman y Dessal (2014) sostienen que las personas sienten descontento y represión, al creer que todas sus acciones profesionales, personales y laborales son medidas dentro de un esquema que promueve acciones de castigo o reprensión por no acogerse a dicha forma de hacer las cosas. Es decir, los contadores declaran que en efecto la empresa les proporciona un estatus, pero el callejón a la aceptación genera dependencia y miedo de no cumplir con los esquemas para ser aceptados. Pero alcanzar el estatus no da la certeza de mantenerlo y no es, necesariamente, para estos profesionales sinónimo de satisfacción o bienestar.

Por otro lado, están las situaciones en las que la empresa recompensa con beneficios diferenciales a los empleados, según el resultado individual o el estatus. Ofrecen acciones en el club, celular, oficina propia, parqueadero o chequeos médicos ejecutivos. Estas recompensas con visos segregacionistas causan incomodidad, porque algunos contadores se sienten excluidos porque los beneficios son solo para aquellos que ostentan determinado estatus.

Para sintetizar, puede decirse que las compañías farmacéuticas (las estudiadas) forjan entre todos sus empleados un estatus social, que debe alcanzarse y, luego, mantenerse. Esto causa sensación de dependencia y miedo a perder el trabajo, los beneficios o el mismo estatus; beneficios que al ser solo para unos, se vuelven discriminatorios.

\section{Políticas basadas en estereotipos}

El estereotipo tiene que ver con un rasgo característico o imaginario que se tiene de un grupo 
social y que, se supone, cualquier miembro del grupo lo posee. Los individuos sujetos de estereotipos negativos afrontan la amenaza de que su comportamiento confirmará tal estereotipo, lo que puede inducir ansiedad (Gómez Vélez y Calderón Carrascal, 2017).

En estas farmacéuticas se observaron dos tipos de políticas fundadas en estereotipos asociadas al malestar laboral: políticas de selección/promoción y políticas de bienestar. Por ejemplo, para la selección estas empresas piden explícitamente profesionales con especializaciones de ciertos países o que hayan hecho sus carreras en determinadas universidades. En esta medida, los procesos de selección restringen la posibilidad de que algunos empleados (quienes no tienen esas características) participen en estos, lo que propicia inconformismo en los sujetos.

Algunos contadores han sufrido frustración debido a que son excluidos en algunos procesos de selección porque no están dentro del "parámetro" determinado. Un ejemplo de las políticas inspiradas en estereotipos se vio cuando un contador participó en un proceso de ascenso y no fue elegido, ya que no cumplía con el estereotipo para el cargo:

En dos oportunidades me dijeron "tú no estás listo, todavía te falta para ser gerente". Pero luego ves en el cargo a una persona con mucha menos experiencia. Me sentí bastante frustrado, porque siento que sí estoy preparado y he tenido retos más grandes, pero ni siquiera te hacen una prueba para saber si tú estás preparado. Simplemente porque no eres de tal universidad o no tienes tal posgrado, te dicen "no te vemos preparado".

Por otro lado, las políticas de bienestar promovidas por las farmacéuticas también fueron analizadas por los contadores; varios de ellos afirman que aunque estas políticas existen, no las pueden disfrutar. Por ejemplo, una de las compañías abordadas en esta investigación promueve una política de FlexTime (tiempo flexible para las horas de ingreso y salida) y otra para uso de gimnasio. Otras políticas de bienestar son medio día libre por motivo del cumpleaños, la fiesta al final del año, vacaciones colectivas o jornadas libres los viernes. Estas políticas están diseñadas de manera general, con la idea de que todos en la empresa pueden hacer uso de ellas. No obstante, el contador no las puede disfrutar, debido a su carga laboral y a sus cronogramas, lo que termina por negarle el disfrute de bienestar. Estas políticas parten del supuesto de que todas las áreas pueden hacer uso de estas; sin embargo, la realidad parece ser otra para los contadores, que por las labores que realizan no pueden tener horarios flexibles; al contrario, sus horarios son extendidos. Aquí el estereotipo se da al definir las políticas con base en una labor o rol que no considera la especificidad del rol contable, al cual sí se le asignan horas de ingreso/salida, entre otras.

En suma, los casos más relevantes de políticas basadas en estereotipos tienen que ver con las políticas de selección/ascenso y con las de bienestar. En este punto el malestar laboral se materializa en la restricción para los contadores de participar en dichas políticas, en que no se considera la especificidad de este rol y en la imposibilidad de disfrutar de los beneficios que brinda la empresa, dada la escasez de tiempo que tienen los contadores y la presión con los plazos en fechas de cierres.

Ahora bien, al analizar los cuatro factores descritos, en función de las causas internas de malestar, se encuentra una leve asociación entre la evaluación del desempeño con el aislamiento y el desempeño a cualquier precio; entre el estatus y el trabajador encerrado; entre las políticas y la pérdida de compromiso. Es decir que los causantes de malestar en otras profesiones parecen tener otra cara, manifestarse de otra manera, para el caso de los contadores. En la etapa de profundización en el análisis de la información, emergieron otros dos factores propios del rol contable: la normatividad y la desterritorialización.

\section{La normatividad}

En Europa, el $20 \%$ de los trabajadores dice tener trastornos de salud asociados al estrés laboral (Dériot, 2010). En Colombia, de acuerdo con Refus, el $38 \%$ de los trabajadores sufre estrés en el trabajo (Portafolio, 2015). Estos datos son considerables y sugieren cuestionamientos para las empresas.

La contabilidad en Colombia está regulada por leyes y múltiples decretos, resoluciones y circulares formuladas por las diferentes entidades estatales, 
como la Superintendencia de Sociedades y la Dirección de Impuestos y Aduanas Nacionales (DIAN), que intervienen, regulan y vigilan la contabilidad. Además, el contador debe estudiar los cambios legislativos que surjan eventualmente, como el proceso de convergencia a las Normas Internacionales de Contabilidad, asociado a la mundialización, y conocer las reformas tributarias.

El malestar laboral relacionado con la normatividad se configura en el hecho de que, además del cumplimiento de las normas locales, los administradores ejercen presión con la planeación tributaria y exigen que el contador logre ser eficiente con los recursos reservados, a la par con el pago de los impuestos. Esto se traduce en un temor a incumplir la norma y a las consecuencias que esto pueda traer.

A lo anterior hay que sumarle que las compañías y sus directivos creen que el contador conoce todas las demás áreas distintas al área financiera; creencia que lleva a que el contador se sobrecargue estudiando temas que no pertenecen a su área. Al respecto, un contador sostiene que "la normatividad local es uno de los grandes látigos del contador $\mathrm{y}$ es que tienes que aprenderte las 10.000 normas $\mathrm{y}$ ahora con el cambio a (IFRS)... siento que la cabeza ya no da para más". Esta sobrecarga produce tensiones a nivel mental y emocional, que se convierten en estrés crónico o sufrimiento.

En este punto, es pertinente distinguir riesgos y factores psicosociales. Los factores psicosociales son las condiciones presentes en el trabajo, que tienen capacidad para afectar al bienestar o a la salud física, psíquica o social (Gómez Vélez y Calderón Carrascal, 2017); comprenden aspectos del medio físico, pero también modos de organización de las tareas, el horario o la demanda de trabajo. Por su parte, los riesgos psicosociales son riesgos de naturaleza y origen diversos, que amenazan la integridad física y el aspecto mental del trabajador; implican aspectos individuales y de interacción con colegas, clientes, etc. Además del temor a incumplir la norma y sus consecuencias (factor), se observan tensiones mentales por la sobrecarga de temas (riesgo) que generan sufrimiento en los contadores.

Por otro lado, existe el denominado cierre contable. Este consiste en cancelar las cuentas de resultados y trasladar dichas cifras a las cuentas de balance respectivas. Esta gestión se hace mensualmente y es material para presentación de informes y declaración de impuestos. Igualmente, no solo es el cierre local (Colombia), sino que los contadores también deben cumplir con otros cierres, ya que se trata de compañías extranjeras.

En la cotidianidad empresarial, es común la presencia de retrasos y errores en la administración de la información. Infortunadamente, el contador debe esperar hasta que todos los movimientos estén completos para iniciar su trabajo; es decir, su labor depende de la labor de otros. El hecho de ser casi el último en el proceso genera presión psicológica, ya que el contador no puede darse plazo para entregar su trabajo: "El cierre es una semana muy estresante para mí. Mi familia sabe que no cuenta conmigo durante esas dos semanas de cierre", comenta uno de los entrevistados. Dicha presión psicológica puede desembocar en estrés laboral.

Es muy probable que algunos aspectos que causan malestar sean comunes a distintas profesiones. En otras palabras, aquello que genera malestar al contador también se lo puede generar al ingeniero o al docente, pero el caso de los contadores y su necesidad de acoplarse a normas, incluso internacionales, es de por sí un tema de interés. En suma, la normatividad asociada al rol del contador en estas farmacéuticas afecta, porque genera presión en cuanto a la eficiencia con los recursos, temor a incumplir la norma, por la creencia que los directivos tienen sobre el conocimiento del contador y porque el proceso contable depende de otros procesos.

\section{La desterritorialización}

Hay quienes consideran que la globalización es clave para el desarrollo económico, a la vez que inevitable e irreversible; otros la ven con hostilidad, incluso temor, debido a que consideran que suscita una mayor desigualdad dentro de cada país y entre los distintos países (Fondo Monetario Internacional, 2000).

Una consecuencia de esto es que las empresas calculan cómo optimizar los recursos de la organización, a la vez que rastrean nuevas vías para sacar provecho de otros mercados. Se desterritorializan procesos y se ejecutan, por partes, en varios países. 
Esta situación ha hecho que las empresas apliquen nuevas prácticas de contratación, como la externalización, el outsourcing y los servicios compartidos (Red Cultural del Banco de la República, 2015). Un ejemplo de estas prácticas y estrategias es el caso de la empresa $\mathrm{P}$, que indicó la expansión de su foco global de servicios financieros en Costa Rica, para contratar a 200 personas en 2017. Actualmente, desde allí 60 empleados atienden a 11 países en Centroamérica y América del Sur (Villalobos Fallas, 2016).

Por lo general, las compañías trasladan procesos y tareas - entre estos la contabilidad-a otros países, buscando trabajar con mano de obra barata. Estas acciones y decisiones causan malestar en los contadores, ya que sienten incertidumbre y miedo por la posibilidad de perder el trabajo. Sobre este particular, un entrevistado señaló:

Estoy entregando el proceso de contabilidad que manejaba. Trasladaron toda el área a otro mercado. Aunque ya me avisaron que trabajo hasta junio, al miedo de quedarme sin trabajo se le suma la tristeza de todo el esfuerzo y los sacrificios por los que tuve que pasar.
La globalización trae cambio y una sensación de inestabilidad (Gaulejac y Hanique, 2015). Los contadores llegan a sentir que su trabajo es fácilmente reemplazado por alguien más, a un costo mucho menor, y esta situación acaba por disparar el malestar laboral, "al pensar que tengo que competir contra otros que están dispuestos a realizar mi trabajo a un precio insignificante", menciona un entrevistado. En estos casos, ni siquiera existe la posibilidad de aceptar un menor salario para quedarse con el puesto; sencillamente, el proceso, que para la empresa es lo importante, se va a otro mercado donde la mano de obra es más barata. Es una muestra de lo que Orejuela (2018) denomina trabajar bajo el régimen de la indiferencia, es decir, hay ausencia total de reconocimiento. No solo no me reconozco en mi trabajo, sino que tampoco me reconocen el trabajo que hago, pues como afirma el anterior entrevistado, se suma "la tristeza de todo el esfuerzo y los sacrificios por los que tuve que pasar". En la figura 1 se exponen los factores generadores de malestar laboral, con sus indicadores y efectos.

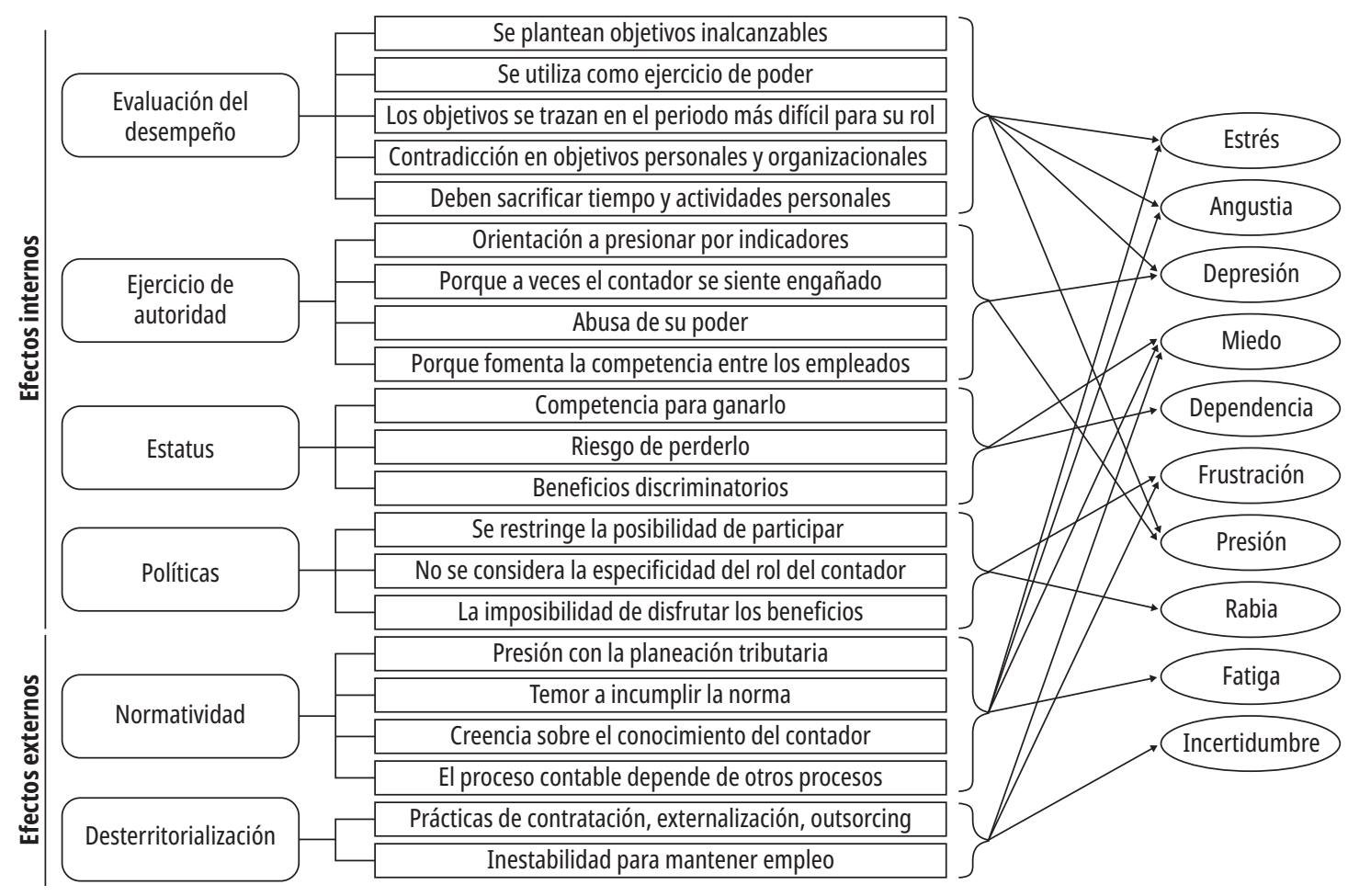

Figura 1. Factores productores de malestar laboral

Fuente: elaboración propia. 


\section{Conclusiones}

Situaciones como el cambio continuo en las políticas tributarias y contables, y el cumplimiento de fechas definidas para los cierres constituyen condiciones que crean malestar laboral. Los estereotipos, desafortunadamente, están presentes en varias de las políticas de recursos humanos de las farmacéuticas, y el malestar en el trabajo se agudiza por la obstinación del grupo ante la negativa de uno de los contadores para realizar lo mismo que todos. Este comportamiento, probablemente discriminatorio, es avivado en sectores de la empresa, situaciones laborales e, incluso, en escenarios tan cotidianas como los almuerzos de trabajo.

Por otra parte, es innegable que la evaluación de desempeño, la autoridad del jefe, la normatividad, etc., constituyen momentos y factores de nerviosismo y ansiedad para los trabajadores, tanto contadores como evaluadores. Esta situación se ve materializada en estados y sentimientos de angustia, estrés, temor, frustración y sufrimiento.

Ahora bien, el estrés puede ser disparado por factores como la evaluación del desempeño, el ejercicio de autoridad o la normatividad; el miedo, por su parte, por la globalización o el estatus social. Esto permite decir que un estado anímico concreto puede ser propiciado por más de un factor a la vez. De igual forma, uno solo de estos cinco factores está favoreciendo más de un efecto a la vez (como se observa en la figura 1). De aquí puede decirse que "después de un siglo, el tiempo de trabajo se ha reducido considerablemente, y la protección de los trabajadores ha progresado en casi todos los sectores. Pero si las condiciones objetivas del trabajo son más confortables, las condiciones subjetivas parecen degradarse" (Gaulejac, 2011, p. 10).

Como se dijo, el tema tratado en la investigación no es un malestar que incapacite, pero no por ello deja de ser malestar. Muchos contadores no lo verbalizan porque expresarlo puede ser asociado con incapacidad para desarrollar el rol o cargo.

\section{Referencias}

Abbagnano, N. (2010). Diccionario de filosofía. Ciudad de México, México: Fondo de Cultura Económica.
Adecco. (2014). Qué tan satisfechos están los colombianos en sus lugares de trabajo. Recuperado de http://adeccocolombia.blogspot.com.co/2014/10/que-tan-satisfechos-estan-los.html

Aubert, N. y De Gaulejac, V. (1991). Le cout de l'excellence. París, Francia: Seuil.

Ávila Toscano, J., Gómez Hernandez, L. y Montiel Salgado, M. (2010). Caracteristicas demográficas y laborales asociadas al síndrome de burnout en profesionales de la salud. Pensamiento Psicológico, 8(15), 39-51.

Barrios, M. e Illada, R. (2013). Valoración del desgaste laboral como riesgo psicosocial. Revista Ingeniería Industrial, 12(1), 69-76.

Bauman, Z. y Dessal, G. (2014). El retorno del péndulo: sobre psicoanálisis y el futuro del mundo líquido. Madrid, España: Fondo de Cultura Económica.

Benaiges, D. (marzo de 2012). Aislamiento social. Revista Verdadera Seducción. Recuperado de http://revista-digital.verdadera-seduccion.com/aislamiento-social/

Bermúdez, H. L. (2017). Sobre la alienación subjetiva en la organización del trabajo actual. Una observación participante en el comercio de la alimentación al detal. Contaduría y Administración, 62(1), 262-278.

Blein Cabestrero, N. (2010). Malestar laboral desde la perspectiva de Grupo Operativo. Cuadernos de temas grupales e institucionales, (14), 1-8.

Bonilla, E. y Rodríguez, P. (2013). Más allá del dilema de los métodos (3ra. Ed.). Bogotá, Colombia: Norma.

Ceballos, J. (1997). Introducción a la sociologia. Santiago de Chile, Chile: LOM Ediciones.

Confederación Regional de Organizaciones Empresariales de Murcia (Croem). (2010). Prevención de riesgos ergonómicos, carga mental: factores de riesgo ergonónico y sus medidas preventivas. Recuperado de http://www. croem.es/prevergo/formativo/4.pdf

Dejours, C. (2003). L'évaluation du travail à l'épreuve du réel: Critique des fondements de l'évaluation. París, Francia: Quae.

Dériot, G. (2010). Rapport d'information. París, Francia: Sénat.

El Espectador. (abril de 2015). Estos son los sectores que mejor pagan en Colombia. Recuperado de https:// www.elespectador.com/noticias/economia/estos-son-los-sectores-mejor-pagan-colombia-articulo-555335

Fernández Collados, M. B. (2012). La doble jornada femenina y sus efectos sobre la salud laboral. Recuperado de http://ve.umh.es/sieg.1/docs/icongresointernacional/ comunicaciones/scv04.pdf 
Fischman. (2015). El estatus de la empresa. Recuperado de https://www.effectusfischman.com/articulos/detalle/62/el-estatus-en-la-empresa

Fondo Monetario Internacional. (abril de 2000). La globalización: ¿amenaza u oportunidad? Recuperado de https://www.imf.org/external/np/exr/ib/2000/es1/041200s.htm

Freud, S. (1988). Obras completas. Buenos Aires, Argentina: Amorrortu.

Galeano, E. (2004). Diseño de proyectos en la investigación cualitativa. Medellín, Colombia: Universidad EAfIT.

Gaulejac, V. de. (2011). Travail, les raisons de la colère. París, Francia: Seuil.

Gaulejac, V. de. y Hanique, F. (2015). Le Capitalisme paradoxant. París, Francia: Seuil.

Gerencia. (junio de 2012). Evaluación del desempeño. Recuperado de http://gerenciadetalentohumano.blogspot. com.co/2012/06/evaluacion-del-desempeno-muchos-son-los.html

Gómez Vélez, M. A. y Calderón Carrascal, P. A. (2017). Salud mental en el trabajo: entre el sufrimiento en el trabajo y la organización saludable. Katharsis, (23), 177-200.

Guerrero Morales, P., Balboa Gallardo, M. y Miranda Hiriart, G. (2017). Sufrimiento y reconocimiento en el trabajo: un estudio de caso. Teuken Bidikay, 8(11), 175-190.

Han, B.-C. (2012). La sociedad del cansancio. Barcelona, España: Herder.

Linhart, D. (2016). Cuando la humanizacion del trabajo enferma a los trabajadores. Teuken Bidikay, 7(8), 25-38.

Loaiza Betancur, E. (2014). El ejercicio de la profesión contable en Colombia. Una mirada desde el paradigma contable del comportamiento basado en la conducta y el deterioro de la salud física y mental. En-contexto, (2), 147-164.

López, F. (2014). Personalidad y estilo de dirección: comportamiento tipo A y acoso laboral. Gestión \& Region, (17), 7-18.

Marulanda, I. (2007). Estrés Laboral. Enemigo silencioso de la salud mental y la satisfacción con la vida. Bogotá, Colombia: Uniandes.

Montes, S. (20 de rebrero de 2019). Ocho de cada diez personas en Colombia están insatisfechas en su empleo. La República. Recuperado de https://www.larepublica. co/alta-gerencia/ocho-de-cada-10-personas-estan-insatisfechas-en-su-empleo-2830069

Morales, R. (noviembre de 2007). Los comportamientos morales en la empresa son ambiguos. Recuperado de
https://www.tendencias21.net/Los-comportamientos-morales-en-la-empresa-son-ambiguos_a1928. html

Naranjo, C. (2012). El liderazgo en la gestion del conocimiento. Manizales, Colombia: Universidad de Manizales.

Orejuela, J. (2018). Clínica del trabajo, el malestar subjetivo derivado de la fragmentación laboral. Medellín, Colombia: EAFIT.

Pedraza, P. (2016). Condiciones generadoras de malestar laboral: "el caso de los contadores públicos en las empresas del sector farmacéutico en Bogota. (Tesis de maestría). Bogotá, Colombia: Universidad Nacional de Colombia.

Portafolio. (agosto de 2015). Secretos para controlar el estrés laboral. Recuperado de http://www.portafolio.co/ tendencias/secretos-controlar-estres-laboral-54546

Red Cultural del Banco de la República. (2015). Globalización. Recuperado de http://www.banrepcultural.org/ blaavirtual/ayudadetareas/economia/globalizacion

Rodríguez, J., De Freitas, S. y Zaá, J. (2012). La contabilidad en el contexto de la globalización y la revolución teleinformática. Revista Venezolana de Análisis de Coyuntura, XVIII(1), 161-183.

Rossano, M., Chatillon, E. y Desmarais, C. (2015). Rupture du contrat psychologique et Risques psycho-sociaux: une RecheRche inteRvention dans le cadre de la théoRie de la conseRvation des Ressources. Revue de gestion des ressources humaines, (95), 58-77.

Sánchez Llull, D., March y Cerdà, M. y Ballester Brage, L. (2015). Malestar social y malestar docente: una investigación sobre el síndrome de desgaste profesional burnout y su incidencia socioeducativa. Aula: Revista de Pedagogía de la Universidad de Salamanca, (21), 245-257.

Semana. (2014). ¿Por qué explotó Transmilenio? Recuperado de http://especiales.semana.com/especiales/ por-que-exploto-transmilenio/index.html

Soler, M. I. (2008). La evaluación de los factores de riesgo psicosocial del trabajo en el sector hortofrutícola: el cuestionario Fapsihos. (Tesis doctoral). Universidad de Murcia, España. Recuperado de http://www.tesisenred.net/handle/10803/11033

Tisnés, H., Lopera, I. C., Pérez, J. D., Ramírez, V. E. y Henao, M. H. (2015). Clínica analítica de las organizaciones. Medellín, Colombia: Fondo Editorial Universidad EAFIT.

Unión General de Trabajadores (UGT). (2014). Salud Ocupacional: Condiciones de Trabajo. Recuperado de 
http://www.iucesmag.edu.co/saludocupacional/articulos/condicionesdetrabajo.pdf

Villalobos Fallas, C. (3 de mayo de 2016). Pfizer amplía su centro de servicios financieros compartido y contrata- rá 200 personas. El Financiero. Recuperado de http:// www.elfinancierocr.com/negocios/Pfizer-servicios-financieros-compartido-contratara_0_950304964.html 